\title{
Asymmetric left ventricular hypertrophy associated with morbid obesity mimicking familial hypertrophic cardiomyopathy
}

Raymond Ching-Chiew $\underline{\text { Wong }}^{1}$, MBBS, MRCP, Kong Bing ${\underline{T a n^{2}}}^{2}$ MBBS, FRCPath

\begin{abstract}
Asymmetric septal hypertrophy with systolic anterior motion of the mitral valve is frequently a phenotypic, but not pathognomonic, expression of genetic hypertrophic cardiomyopathy (HCM) with or without obstruction. It can, however, be associated nonspecifically with other forms of increased left ventricular (LV) afterload. We herein report the case of a young man with obesity cardiomyopathy and heart failure who presented with asymmetric septal hypertrophy and marked LV hypertrophy, and endomyocardial biopsy ruled out genetic HCM.
\end{abstract}

Keywords: asymmetric left ventricular hypertrophy, endomyocardial biopsy, familial hypertrophic cardiomyopathy, morbid obesity

\section{INTRODUCTION}

Obesity is associated with increased myocardial wall thickness and varying degrees of diastolic dysfunction. ${ }^{(1,2)}$ The proposed mechanism for the observed myocardial changes is an increase in the total circulating blood volume because of a higher metabolic demand for adipose tissues. ${ }^{(3)}$ Cardiomyopathy in obesity usually presents as dilated cardiomyopathy (DCM) due to eccentric remodelling. ${ }^{(2,4)}$ DCM is an independent predictor of heart failure. ${ }^{(5)}$ On another note, asymmetric septal hypertrophy with systolic anterior motion of the mitral valve is frequently a phenotypic, but not pathognomonic, expression of genetic hypertrophic cardiomyopathy (HCM) with or without obstruction. We herein report the case of a morbidly obese man who presented with congestive heart failure (CHF).

\section{CASE REPORT}

Our patient was a morbidly obese 29-year-old man who weighed $149 \mathrm{~kg}$ and had a body mass index (BMI) of $40 \mathrm{~kg} / \mathrm{m}^{2}$ at presentation. He was previously diagnosed with $\mathrm{HCM}$ at 12 years of age, but was subsequently lost to follow-up. In 2010, he developed bilateral deep vein thromboses, where the diagnosis of protein $\mathrm{S}$ deficiency was made. He was put on long-term anticoagulation, but lacked compliance to medication. In November 2012, he complained of breathlessness that was made worse on exertion. A clinical diagnosis of $\mathrm{CHF}$ was made. Echocardiography showed marked asymmetric septal hypertrophy (septal wall thickness and posterior wall thickness measured $23 \mathrm{~mm}$ and $14 \mathrm{~mm}$, respectively; the septal to posterior wall thickness ratio was 1.64), systolic anterior motion of the mitral valve, moderately severe posteriorly-directed mitral regurgitation, and pulmonary artery systolic pressure of $69 \mathrm{mmHg}$. Left ventricular (LV) systolic function was hyperdynamic, and the maximum pressure gradient across the LV outflow tract was 57 mmHg (Figs. 1 \& 2). There was no 'ground glass' appearance of the myocardium. Diastolic dysfunction was evident based on the presence of restrictive filling pattern (mitral E/A ratio of 2.15 and short mitral deceleration time of $158 \mathrm{~ms}$ ) and elevated mitral E/E' ratio of 15.6 and 18.7 at the septal and lateral mitral annuli, respectively, indicating high filling pressures (Fig. 3). However, these findings are nonspecific and may be widely observed in familial HCM. A diagnosis of diastolic CHF and obstructive HCM was made. Notably, he had a similar presentation at another hospital, ten months before presenting at our institution.

At presentation to our institution, the patient responded to diuretic and noninvasive positive pressure ventilation. He underwent a right heart study that confirmed the diagnosis of severe pulmonary hypertension (the mean pulmonary artery pressure was $61 \mathrm{mmHg}$ ), although the pulmonary capillary wedge pressure was elevated at $41 \mathrm{mmHg}$. Systemic blood pressure was $120 / 87 \mathrm{mmHg}$. The ensuing endomyocardial biopsy taken from the right ventricular septum showed nonspecific, mildly hypertrophied myocytes with diameters ranging from $20 \mu \mathrm{m}$ to $30 \mu \mathrm{m}$ and containing enlarged nuclei. Neither obvious myofibre disarray nor small intramural coronary arteriole dysplasia was observed (Fig. 4).

The final diagnosis was diastolic CHF and asymmetric LV hypertrophy contributed to by morbid obesity and possibly obstructive sleep apnoea, where the cardiac morphology mimicked familial hypertrophic obstructive cardiomyopathy (HOCM).

\section{DISCUSSION}

Obesity is associated with increased myocardial wall thickness, larger LV mass index by height, and diastolic dysfunction, ${ }^{(1)}$ as evidenced by the impairment of diastolic LV longitudinal function and increased LV diastolic filling pressure. ${ }^{(6)}$ Cardiomyopathy in obesity can present as DCM, ${ }^{(4,7)}$ although this finding has been disputed. ${ }^{(8)}$ In obese patients associated with DCM, myocardial structural changes can be insignificant even at the ultrastructural level. ${ }^{(4)}$ 'Obesity cardiomyopathy' has been loosely defined as clinical heart failure directly associated with myostructural

1Department of Cardiology, National University Heart Centre, 2Department of Pathology, National University of Singapore, Singapore

Correspondence: Dr Raymond Ching-Chiew Wong, Senior Consultant, Department of Cardiology, National University Heart Centre, 1E Kent Ridge Road, NUHS Tower Block, Level 9, Singapore 119228. raymond_cc_wong@nuhs.edu.sg 

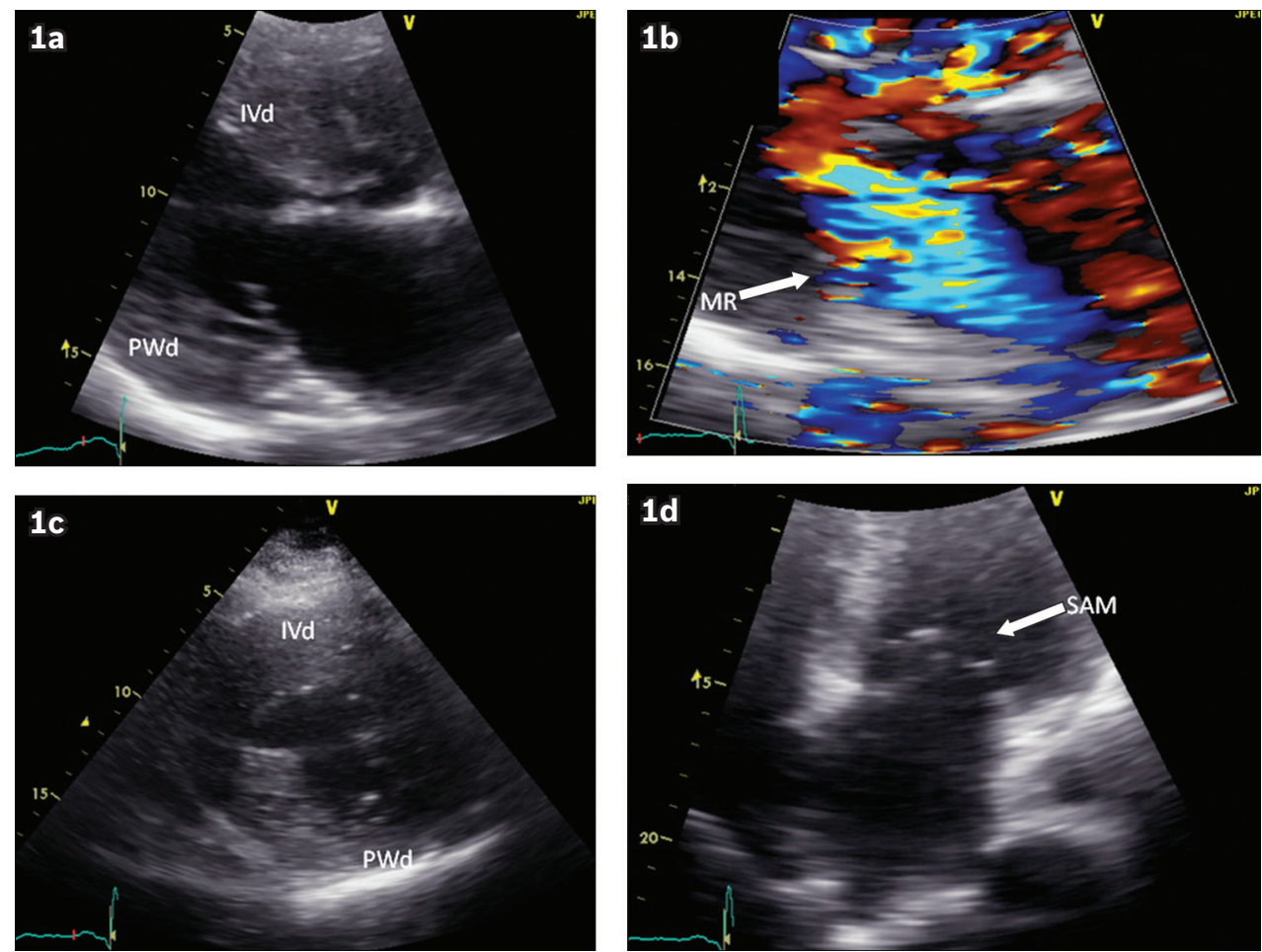

Fig. 1 Transthoracic echocardiogram of the patient. Parasternal long axis view shows (a) thickened interventricular septum in diastole (IVd) measuring $23 \mathrm{~mm}$ and a mild thickened posterior wall in diastole (PWd) measuring $14 \mathrm{~mm}$, and (b) moderately severe posteriorly-directed mitral regurgitation (arrow). (c) Parasternal short axis view shows markedly thickened interventricular septum during diastole (IVd) and a mild thickened posterior wall (PWd), with a ratio of 1.64. (d) Apical four-chamber view shows systolic anterior motion (SAM) of the mitral leaflet during systole (arrow).

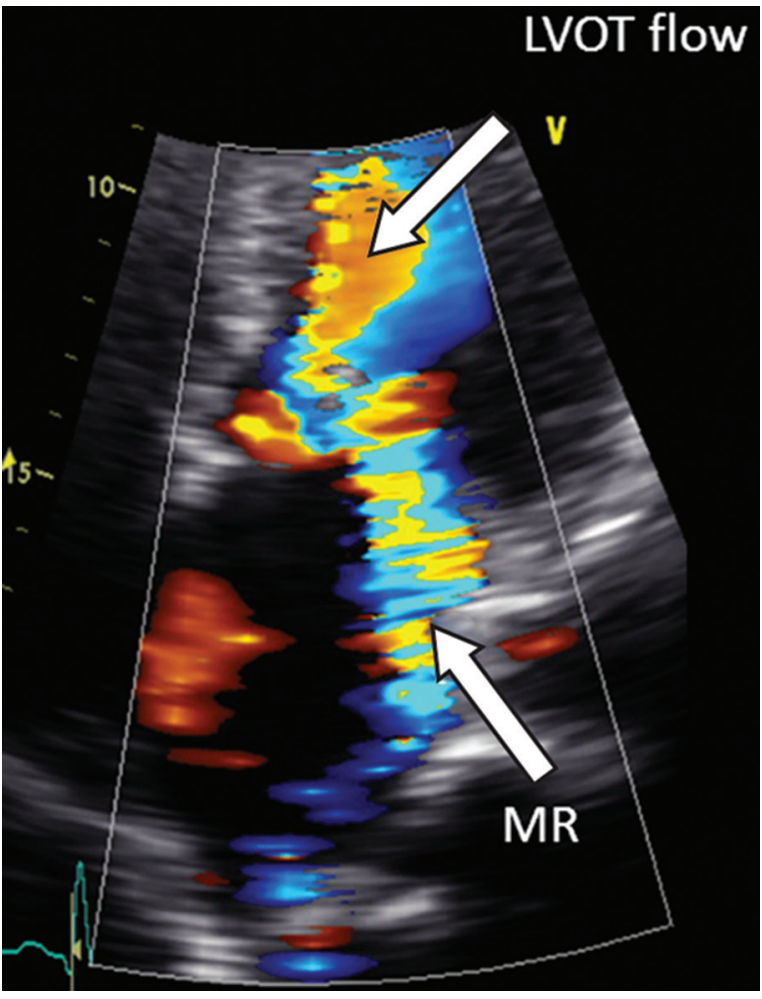

Fig. 2 Apical four-chamber echocardiogram view with colour flow imaging shows flow acceleration across the left ventricular outflow tract and eccentric mitral regurgitation directed posterolaterally.

changes in a morbidly obese person. Indeed, morbid obesity is an independent predictor of heart failure. ${ }^{(5)}$ There is, thus far, no literature report of obesity cardiomyopathy presenting with asymmetric septal hypertrophy and systolic anterior motion phenotype, especially in the absence of increased afterload such as marked hypertension. Preload reserve is increased in obese patients, as demonstrated by the significant increase in the left atrial dimension. ${ }^{(6)}$

In our young patient, diabetes mellitus was ruled out based on normal random and fasting blood glucose profile. His recurrent diastolic CHF presentation is highly unusual for a patient with genetic $\mathrm{HCM}$ who has neither atrial fibrillation nor a reduced left ventricular ejection fraction $<50 \%$ (i.e. end-stage phase of $\mathrm{HCM}$ ). Histological investigation revealed nonspecific LV hypertrophy, as evidenced by increased myofibre dimensions and connective tissue deposition, albeit the appearance of a classic HOCM cardiac morphology on echocardiography and chest computed tomography (Fig. 5).

Kasper et $\mathrm{al}^{(7)}$ compared 43 obese with 409 nonobese heart failure patients. While specific aetiologies were found in two-thirds of the nonobese patients, significantly fewer (about a quarter) of the obese cohort had identifiable heart failure risk factors. The most common finding from endomyocardial biopsy in the obese group was mild myocyte hypertrophy $(67 \%)$. Notably, BMl correlated positively with right heart pressures and cardiac output, pulmonary vascular resistance index and systolic blood pressure. A significantly higher proportion of obese patients were found to have idiopathic DCM, compared with nonobese patients. ${ }^{(7)}$ Using ultrasonic integrated backscatter (IBS) in assessing myocardial reflectivity (index of elevated myocardial collagen content), Di Bello et al(6) 

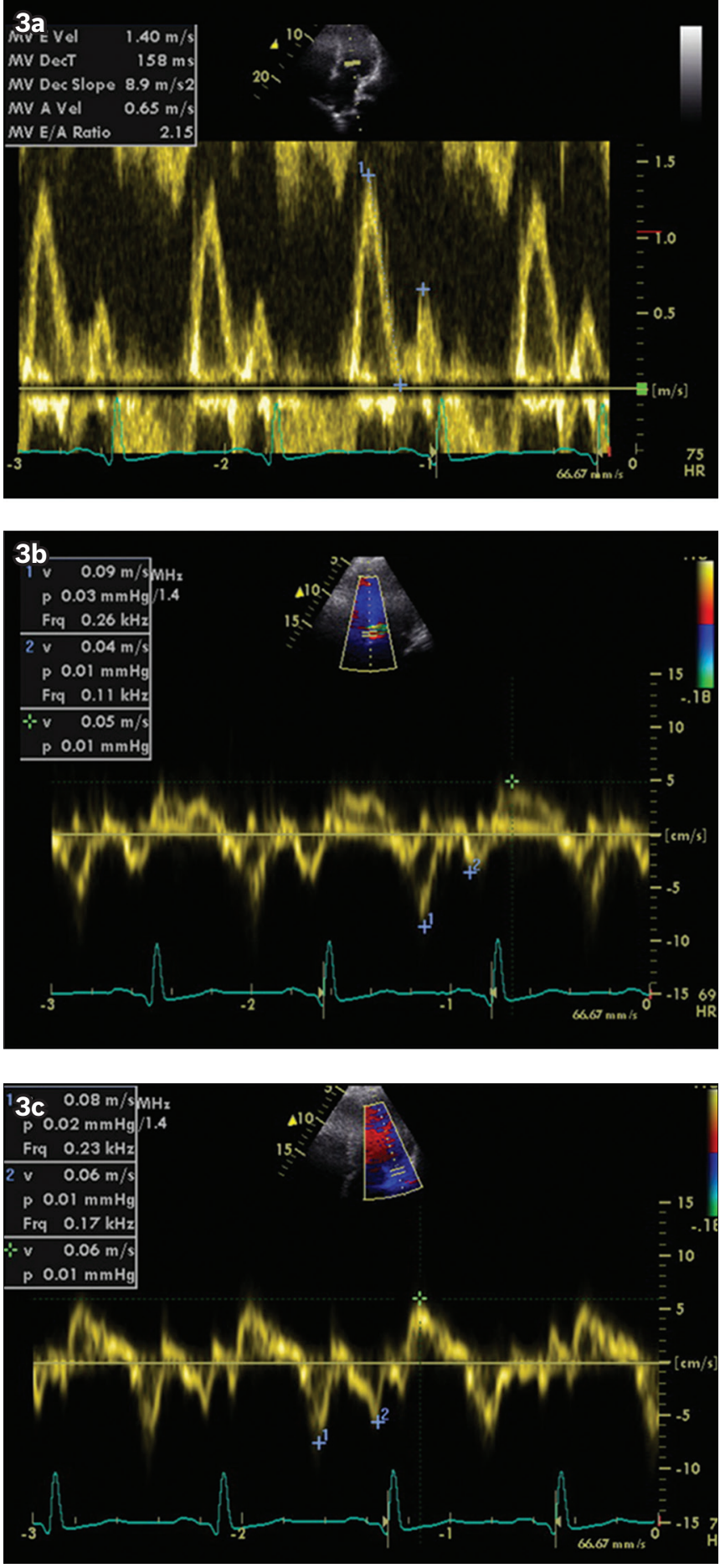

Fig. 3 (a) Apical four-chamber view shows mitral inflow velocities. E and A wave velocities measured 140 and 65 millisec, respectively, giving E/A ratio of 2.1. No L wave (mid-diastolic) was observed. (b \& c) Tissue Doppler echocardiogram shows E' velocities measuring $9 \mathrm{~cm} / \mathrm{s}$ and $7.5 \mathrm{~cm} / \mathrm{s}$ at the septal and lateral mitral annuli, respectively, giving a E/E' ratio of 15.6 and 18.7 , respectively.

showed that obese patients had higher IBS indexes at the septum level than in nonobese patients (i.e. the control group), indicating fibrosis-forming processes. Obese patients also had significantly lower cyclic variation index both at the septum and posterior wall. ${ }^{(6)}$ The study therefore suggested that obese patients exhibit myocardial structural and functional alterations that are physiologically consistent with incipient or subclinical obesity cardiomyopathy.

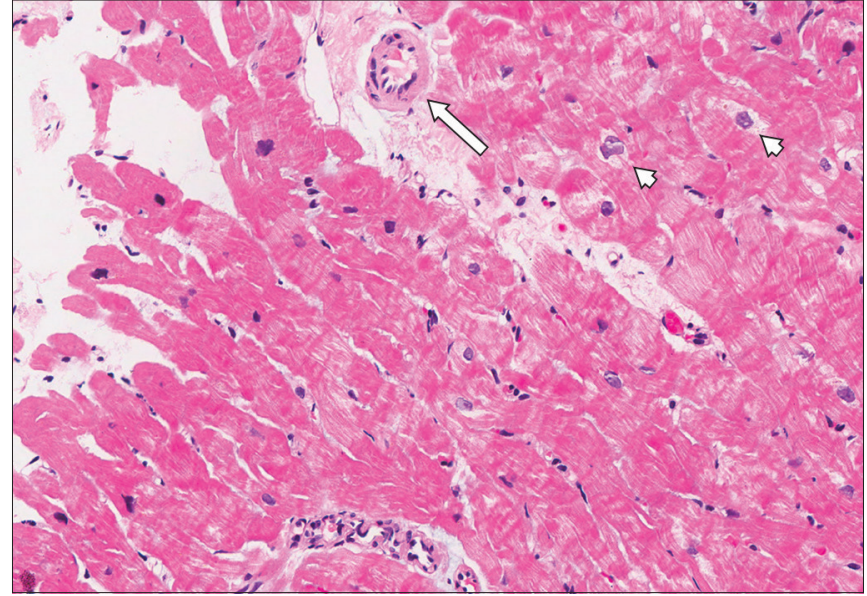

Fig. 4 Photomicrograph of the myocardium shows hypertrophied myofibres with enlarged nuclei (arrowheads), with no evidence of myofibril architectural disarray, and the intramural arterioles appeared normal (arrow) (Haematoxylin \& eosin, x 200).

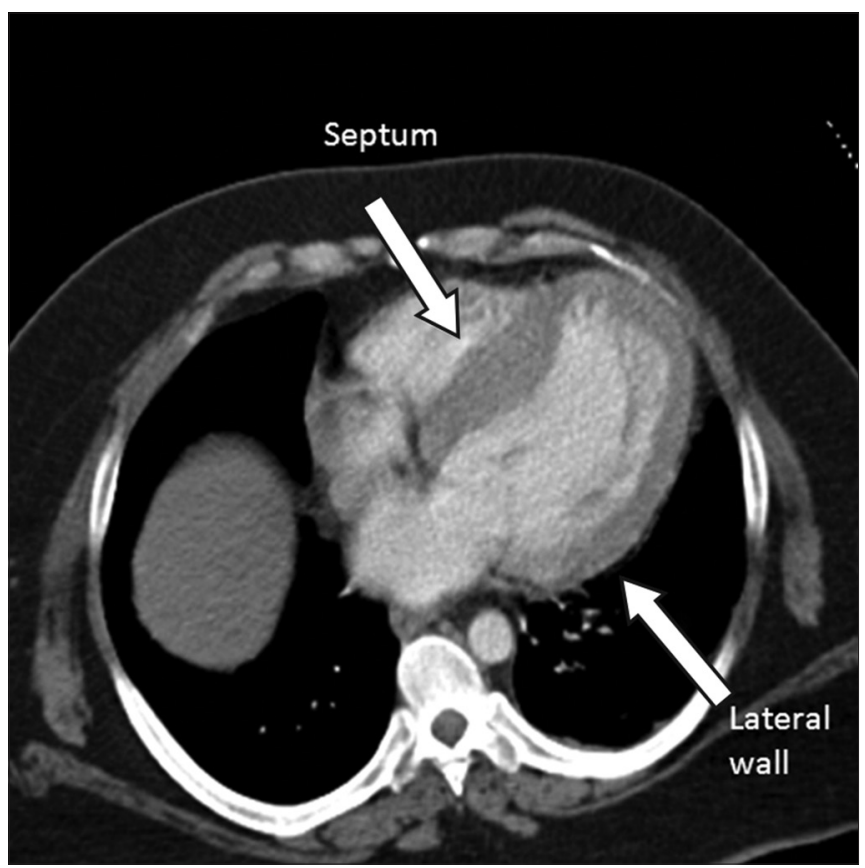

Fig. 5 Chest CT scan shows four-chamber of the heart. The septal and lateral wall thicknesses are $26 \mathrm{~mm}$ and $12.8 \mathrm{~mm}$, respectively, with a resulting septal-to-posterior wall thickness ratio of 2.1 .

Additionally, in our young obese patient, it may well be that his diastolic heart failure was precipitated by altered physiological mechanism in the context of obstructive sleep apnoea, which is highly associated with heart failure, ${ }^{(9)}$ chronic circulatory volume overload $^{(10)}$ and systemic inflammation with elevated concentrations of plasma adipokines from excess adipose tissue and cytokines. ${ }^{(11)}$

In conclusion, this case report highlights the importance of endomyocardial biopsy in patients with HCM with unusual presentation. Aggressive weight loss strategy including bariatric surgery may be effective in reversing LV hypertrophy. ${ }^{(12)}$

\section{REFERENCES}

1. Mehlman E, Bright JM, Jeckel K, et al. Echocardiographic evidence of left ventricular hypertrophy in obese dogs. J Vet Intern Med 2013; 27:62-8. 
2. Wong C, Marwick TH. Obesity cardiomyopathy: diagnosis and therapeutic implications. Nat Clin Pract Cardiovasc Med 2007; 4:480-90.

3. Alpert MA, Fraley MA, Birchem JA, Senkottaiyan N. Management of obesity cardiomyopathy. Expert Rev Cardiovasc Ther 2005; 3:225-30.

4. Saito T, Asai K, Sato S, et al. Myocardial alterations and clinical implications associated with recovery of cardiac function in dilated cardiomyopathy with obesity. Int J Cardiol 2013; 168:144-50.

5. Kenchaiah S, Evans JC, Levy D, et al. Obesity and the risk of heart failure. N Engl J Med 2002; 347:305-13.

6. Di Bello V, Santini F, Di Cori A, et al. Obesity cardiomyopathy: is it a reality? An ultrasonic tissue characterization study. J Am Soc Echocardiogr 2006; 19:1063-71.

7. Kasper EK, Hruban RH, Baughman KL. Cardiomyopathy of obesity: a clinicopathologic evaluation of 43 obese patients with heart failure. Am
J Cardiol 1992; 70:921-4.

8. Khan MF, Movahed MR. Obesity cardiomyopathy and systolic function: Obesity is not independently associated with dilated cardiomyopathy. Heart Fail Rev 2013; 18:207-17.

9. Gottlieb DJ, Yenokyan G, Newman AB, et al. Prospective study of obstructive sleep apnea and incident coronary heart disease and heart failure: the sleep heart health study. Circulation 2010; 122:352-60.

10. Kaltman AJ, Goldring RM. Role of circulatory congestion in the cardiorespiratory failure of obesity. Am J Med 1976; 60:645-53.

11. Frankel DS, Vasan RS, D'Agostino RB Sr, et al. Resistin, adiponectin, and risk of heart failure the Framingham offspring study. J Am Coll Cardiol 2009; 53:754-62.

12. Ristow B, Rabkin J, Haeusslein E. Improvement in dilated cardiomyopathy after bariatric surgery. J Card Fail 2008; 14:198-202. 